\title{
The inhibitory role of miR-485-5p in colorectal cancer proliferation and invasion via targeting of CD147
}

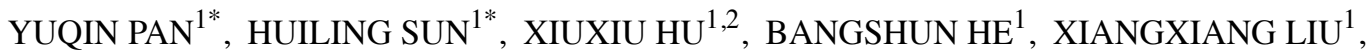 \\ TAO XU ${ }^{1}$, XIAOXIANG $\mathrm{CHEN}^{1,2}$, KAIXUAN ZENG ${ }^{1,2}$ and SHUKUI WANG $^{1,2}$ \\ ${ }^{1}$ Central Laboratory, Nanjing First Hospital, Nanjing Medical University, Nanjing, Jiangsu 210006; \\ ${ }^{2}$ Clinical Medical College, Southeast University, Nanjing, Jiangsu 210009, P.R. China
}

Received August 10, 2017; Accepted February 8, 2018

DOI: $10.3892 /$ or.2018.6309

\begin{abstract}
Recently, miR-485-5p was identified as a tumor-suppressing microRNA in some human cancers. However, the expression and biological role of miR-485-5p in colorectal cancer (CRC) remain unclear. Herein, we found that the expression of miR-485-5p in CRC tissues and cell lines was much lower than that in the control, respectively. Moreover, the overexpression of miR-485-5p could inhibit the proliferation and invasion of CRC cell lines in vitro and in vivo. Furthermore, we determined that miR-485-5p could decrease the expression of CD147 by directly targeting CD147 3'UTR. In addition, the expression of CD147 was inversely correlated with the expression of miR-485-5p in CRC tissues. Collectively, our results revealed that miR-485-5p played a pivotal tumor-suppressing role in CRC through downregulation of cancer-associated gene CD147, and may provide a better understanding of the pathogenesis in CRC and a new bio-target for CRC therapy.
\end{abstract}

\section{Introduction}

The 2017 Cancer Statistics revealed that colorectal cancer (CRC) was the third most common cancer in the US (1). The common causes of CRC-related deaths include late tumor diagnosis and rapid progression. Despite substantial progress achieved in interventional radiology, surgery, regional and systemic therapy for CRC in recent years, the overall 5 -year survival rate remains unsatisfactory (2). Population screening programs for identification of patients with early

Correspondence to: Professor Shukui Wang or Mrs. Yuqin Pan, Central Laboratory, Nanjing First Hospital, Nanjing Medical University, 68 Changle Road, Nanjing, Jiangsu 210006, P.R. China E-mail: shukwang@163.com

E-mail: panyuqin01@163.com

*Contributed equally

Key words: miR-485-5p, tumor-suppressing, colorectal cancer, CD147, pathogenesis
CRC stages is important for increasing the survival of patients. At present, the precise mechanisms of carcinogenesis in CRC are still unknown. Therefore, seeking novel diagnostic biomarkers and therapeutic strategies is necessary and urgent for the early detection of CRC before the onset of metastasis in order to improve the survival rate of patients with CRC.

MicroRNAs (miRNAs) are a class of small-non-coding RNA molecules (-18-25 nucleotides in length). It has been demonstrated that miRNAs could regulate gene expression at the post-transcriptional level, and regulate several cellular biological processes, such as apoptosis, proliferation and migration. It has been reported that dysregulated miRNAs may play important roles in the occurrence and development of CRC (3-5). Presently, circulating miRNAs could be stably detected in plasma or serum, as well as in other body fluids $(6,7)$. Therefore, miRNAs may act as potential biomarkers for diagnostic and prognostic evaluation of CRC. In recent years, miR-485-5p has received much attention as a functional miRNA. Previous studies reported that miR-485-5p was significantly downregulated and overexpression of miR-485-5p could suppress cell proliferation, metastasis and promote cell apoptosis in many cancers (8-11). Thus, miR-485-5p may function as a potential tumor suppressor. However, the expression of miR-485-5p in CRC and its role in CRC carcinogenesis are unknown.

Cluster of differentiation 147 (CD147) which belongs to the immunoglobulin superfamily (IgSF) is a glycosylated cell surface transmembrane protein, also known as basigin (BSG). Previous studies have demonstrated that aberrant expression of CD147 was observed in many cancers, and the increased expression of CD147 in CRC was associated with poor prognosis (12-18). In our previous studies, we found that CD147 significantly contributed to tumor growth and metastasis in CRC $(19,20)$.

In the present study, we confirmed the differential expression of miR-485-5p in CRC cancer tissues and CRC cell lines, and demonstrated that miR-485-5p could regulate cell proliferation, migration and invasion by targeting CD147 in vitro. miR-485-5p may play an important role in the pathogenesis of $\mathrm{CRC}$ as a tumor suppressor, and serve as a potential diagnostic and therapeutic target in CRC. 


\section{Materials and methods}

Clinical tissue specimens. Forty-seven pairs of CRC tissue specimens and adjacent non-tumor (ANT) tissues were obtained from Nanjing First Hospital Affiliated to Nanjing Medical University. The tumor tissues and non-tumor tissues were snap-frozen following surgery and immediately stored at $-80^{\circ} \mathrm{C}$ until use. The present study was approved by the Medical Ethics Committee of Nanjing First Hospital Affiliated to Nanjing Medical University, and all patients provided written informed consents. Immunohistochemistry was performed using Histostain ${ }^{\circledR}$-SP kits (Invitrogen; Thermo Fisher Scientific, Inc., Carlsbad, CA, USA) according to the manufacturer's instructions. Immunopositivity was evaluated independently by two pathologists.

Plasmid constructs and generation of stable cell clones. To construct the miR-485-5p overexpression vector, the miR-485-5p precursor_RNA ( 85 bp) was synthesized by Synbio Technologies (Suzhou, China), and then cloned into pcDNA3.1(+) in NheI and EcoR sites (Invitrogen; Thermo Fisher Scientific, Inc.), and named pcDNA3.1-miR-485-5p. The anti-miR-485-5pand anti-miR-NC were synthesized by RiboBio Co.,Ltd.,(Guangzhou,China). We used TargetScan (http://www .targetscan.org/) to find the predicted target of miR-485-5p. The sequence alignment confirmed that the seed sequence of miR-485-5p was complementary to the 3'UTR of CD147 (BSG) in positions 229-257, 429-463 and 510-534. The wild-type and mutant 3'untranslated region (UTR) (822 bp) of target gene BSG (CD147) were synthesized by Synbio Technologies, and then cloned into pmirGLO in NheI and SalI sites, named CD147/3'UTR-WT and CD147/3'UTR-Mut. To construct the CD147 expression vector, the CD147 DNA (828 bp) was synthesized by Synbio Technologies, and then cloned into pcDNA3.1(+) in NheI and XhoI sites (Invitrogen; Thermo Fisher Scientific, Inc.), and named pcDNA3.1-CD147. All constructs were further confirmed by sequencing.

Cell lines and culture conditions. Cell lines HCT116, HT29, HCT8 (human colon cancer cell lines) and FHC (normal human intestinal epithelial cell line) were obtained from Shanghai Cell Collection, Chinese Academy of Sciences. All aforementioned cell lines were maintained in Dulbecco's modified Eagle's medium (DMEM; Hyclone; GE Healthcare Life Sciences, Logan, UT, USA). The cells were incubated in a humidified incubator at $37^{\circ} \mathrm{C}$ with $5 \% \mathrm{CO}_{2}$.

RNA extraction and real-time quantitative PCR (RT-qPCR). Total RNA was isolated from tissues and cell lines using TRIzol reagent (Invitrogen; Thermo Fisher Scientific, Inc.) according to the manufacturer's instructions. Reverse transcription was performed using Prime-Script RT reagent kit (Takara Bio, Inc., Otsu, Japan). Quantitative PCR was performed on the cDNA using specific primers (Sangon, Shanghai, China) for CD147 and $\beta$-actin (as an internal control), which are listed in Table I. The expression of miR-485-5p was detected by Stem-loop reverse transcriptase polymerase chain reactions (RT-PCR). The primers for miR-485-5p and U6 snRNA were purchased from RiboBio Co., Ltd. All reactions were carried out on the Applied Biosystems 7500 Sequence Detection
System (Applied Biosystems, Foster City, CA, USA). Relative expression levels were calculated as ratios normalized against those of $\beta$-actin or U6 snRNA. Comparative quantification was determined using the $2^{-\Delta \Delta \mathrm{Ct}}$ method. Each sample was prepared in triplicate and all reactions were triplicated independently to ensure the reproducibility of the results.

Stable cell line generation. The transfected CRC cells in six-well plates were selected with G418 $(1,000 \mu \mathrm{g} / \mathrm{ml})$. Two weeks later, few cells survived, and G418 was reduced to $500 \mu \mathrm{g} / \mathrm{ml}$. A stable cell line which could stably express miR-485-5p was established, and the expression of miR-485-5p was assessed by Stem-loop RT-PCR.

CCK- 8 assay. Cell growth viability was assessed using Cell Counting Kit-8 (CCK-8) (Beyotime Institute of Biotechnology, Shanghai, China). The cells were seeded in a 96-well plate at a density of $1 \times 10^{4}$ cells/well and incubated for $24,48,72$ and $96 \mathrm{~h}$, respectively. Following the addition of $10 \mu \mathrm{l}$ of CCK-8 into the medium, each plate was assessed at $450 \mathrm{~nm}$ using the microplate reader Tecan M200 PRO (Tecan Austria GmbH, Untersbergstr, Austria).

In vitro invasion assay. For the invasion assays, Matrigel (BD Biosciences, Franklin Lakes, NJ, USA) was polymerized in Transwell chambers for $45 \mathrm{~min}$ at $37^{\circ} \mathrm{C}$. After the Matrigel was solidified, $1 \times 10^{5}$ transfected CRC cells were seeded in the top chamber with serum-free medium, and the bottom chamber was filled with DMEM containing 10\% FBS.

After $24 \mathrm{~h}$, the cells on the lower surface of the membrane were fixed with alcohol and stained with crystal violet, and then the number of cells were counted under a light microscope. Each assay was carried out in triplicate and repeated three times.

In vivo proliferation assay. All BALB/c nude mice were purchased from the Comparative Medical Center of Yangzhou University and maintained under specific pathogen-free conditions. The animal experiments were approved by the Animal Care Committee of Nanjing Medical College (acceptance no. SYXK20160006). The miR-485-5p transfected HT29 cells were trypsinized to a single cell suspension, and then subcutaneously injected into the flank area of adult (6-8-week-old) athymic male nude mice. The developed tumors were examined weekly and measured in two dimensions, and the tumor volume was calculated according to the formula: (width) ${ }^{2} \mathrm{x}$ length $x 0.5$. The mice were euthanized 42 days post-inoculation. Animal experiments were performed in accordance with the Institutional Guidelines for Animal Care by the Nanjing Medical University, Jiangsu, China.

Luciferase assay. The 293T and HT29 cells were seeded in 96-well plates, respectively. Co-transfection with either the empty vector or pcDNA3.1-miR-485-5p and the luciferase reporter comprised of the 3'UTR of CD147,CD147/3'UTR-NC or CD147/3'UTR-Mut, using Lipofectamine 2000 (Invitrogen-Life Technologies, CA, USA) was performed. After $16 \mathrm{~h}$ the old medium was removed and DMEM which contained 5\% FBS was added. Then, the cells were harvested $48 \mathrm{~h}$ after transfection and luciferase activity was assessed as chemiluminescence using the Dual-Luciferase reporter assay system 
Table I. Primers of CD147 and $\beta$-actin for real-time PCR.

\begin{tabular}{ll}
\hline Target & \multicolumn{1}{c}{ Primers } \\
\hline CD147 & $\begin{array}{l}\text { Sense: 5'-CCATGCTGGTCTGCAAGTCAG-3' } \\
\text { Antisense: 5'-CCGTTCATGAGGGCCTTGTC-3' }\end{array}$ \\
\multirow{2}{*}{-actin } & $\begin{array}{l}\text { Sense: 5'-CTGGAACGGTGAAGGTGACA-3' } \\
\text { Antisense: 5'-AAGGGACTTCCTGTAACAACGCA-3' }\end{array}$
\end{tabular}

(Tecan Austria GmbH, Untersbergstr, Austria) according to the manufacturer's protocol.

Western blot analysis. The transfected CRC cells were harvested and lysed by three cycles of freeze/thaw at $-80^{\circ} \mathrm{C}$. Total protein was separated by $10 \%$ SDS-PADE gels, and transferred to a polyvinylidene difluoride (PVDF) membrane. The membrane was blocked with $5 \%$ skimmed milk powder (soluble in TBST buffer solution) at $4^{\circ} \mathrm{C}$ for $2 \mathrm{~h}$, and then the membrane was incubated with mouse anti-CD147 primary antibodies (1:5,000; cat. no. Ab108317) and GAPDH (1:10,000; cat. no. ab181602,) at room temperature for $2 \mathrm{~h}$, followed by secondary antibodies goat anti-rabbit IgG-HRP (1:10,000; cat. no. Ab6721; Abcam, Inc., Cambridge, UK) for $1 \mathrm{~h}$ at room temperature. The proteins were visualized by ECL detection system (GE ImageQuant LAS 4000; GE Healthcare Bio-Sciences, Uppsala, Sweden).

Statistical analysis. The SPSS 15.0 software (SPSS, Inc., Chicago, IL, USA) was used for general statistical. Experimental data are presented as the mean \pm standard deviation (SD) and analyzed by Student's t-test or one-way analysis of variance (ANOVA), and the criterion for statistical significance was considered to be $\mathrm{P}<0.05$.

\section{Results}

miR-485-5p is downregulated in CRC tissues and cell lines. To identify the expression of miR-485-5p in the CRC, forty-seven pairs of CRC and ANT tissues were assessed by RT-qPCR. As shown in Fig. 1A, the expression levels of miR-485-5p were downregulated in CRC tissues compared with ANT tissues $(\mathrm{P}<0.05)$. The miR-485-5p expression in CRC cell lines (HCT116, HT29, HCT8) were also detected and compared to the level of normal human intestinal epithelial cell line (FHC). As shown in Fig. 1B, the expression levels of miR-485-5p in all CRC cell lines (HCT116, HT29, HCT8) were lower than that of the FHC cell line, and miR-485-5p expression in the HT29 cells was relatively the lowest.

miR-485-5p inhibits CRC cell proliferation and invasion in vitro. We then investigated the effects of miR-485-5p on cell proliferation and invasion in CRC cells. First, we transfected miR-485-5p overexpression vector pcDNA3.1-miR-485-5p into HCT116, HT29 and HCT8 cells to increase miR-485-5p expression, and the stably transfected cell lines were separated by $\mathrm{G} 418$. The results revealed that the expression of miR-485-5p in CRC cells transfected with the miR-485-5p overexpression vector was increased compared with the control transfected cells, respectively ( $\mathrm{P}<0.05$, Fig. 2). Second, we performed
A
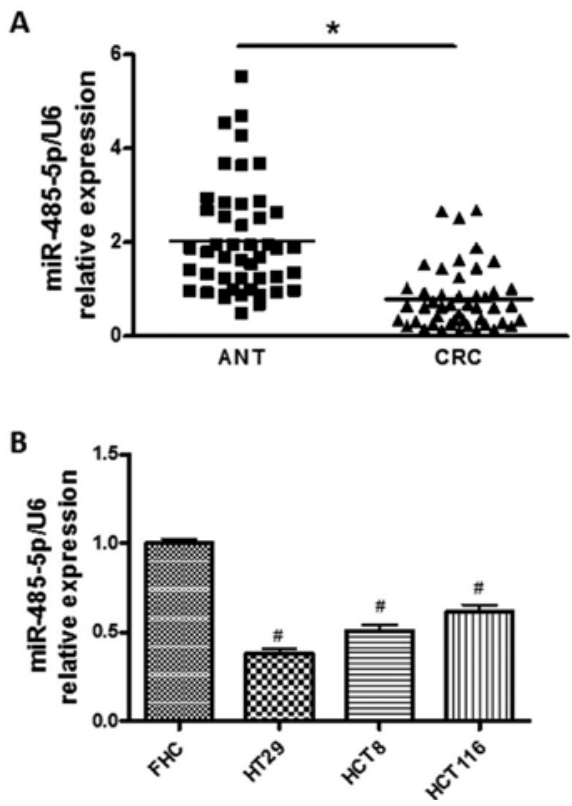

Figure 1. Relative miR-485-5p expression in CRC tissues and cell lines. (A) The expression of miR-485-5p was analyzed by qRT-PCR in CRC tissues compared with ANT tissues, ${ }^{*} \mathrm{P}<0.05$. (B) The expression of miR-485-5p was analyzed in CRC cell lines and FHC cells by qRT-PCR. " $\mathrm{P}<0.05$. CRC, colorectal cancer; ANT, adjacent non-tumors.

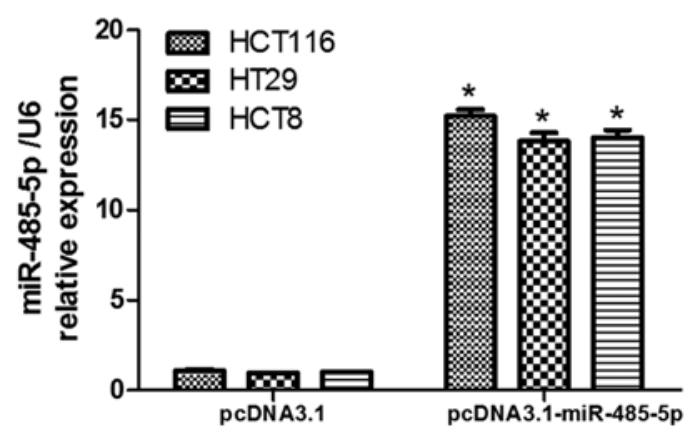

Figure 2. Expression of miR-485-5p was detected by qRT-PCR in transfected CRC cells. The expression of miR-485-5p in HCT116, HT29 and HCT8 cells which were transfected with pcDNA3.1-miR-485-5p was significantly upregulated compared with the control groups, respectively, ${ }^{*} \mathrm{P}<0.05$. CRC, colorectal cancer.

CCK- 8 assays in transfected CRC cells, and the results revealed that the proliferation of CRC cells transfected with miR-485-5p overexpression vector was significantly decreased compared with the control transfected cells $(\mathrm{P}<0.05$, Fig. 3$)$. Moreover, the results of the cell invasion assay revealed that overexpression of miR-485-5p could reduce the invasion rate of HT29, HCT116 and HCT8 cells compared with the control, respectively (Fig. 4).

miR-485-5p inhibits CRC tumor growth in vivo. To investigate whether the overexpression of miR-485-5p could inhibit CRC development in vivo, we established the CRC xenograft models with transfected HT29 cells. The results revealed that overexpression of miR-485-5p could significantly inhibit the growth of tumors during the same observation period compared with the control group in HT29 xenograft models, as shown in Fig. 5A and B. 

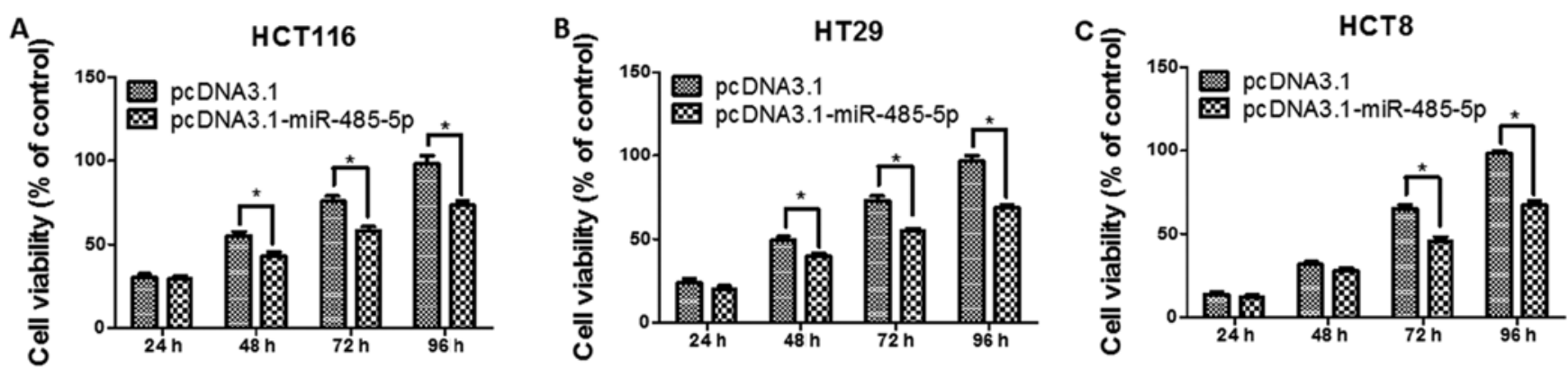

Figure 3. miR-485-5p suppresses cell proliferation of CRC cells. The proliferation of transfected cells (HCT116, HT29 and HCT8) was examined by CCK-8 assay at $24,48,72$ and $96 \mathrm{~h}$. (A) The proliferation of HCT116 cells transfected with pcDNA3.1-miR-485-5p. Overexpression of miR-485-5p was significantly downregulated compared with the control group after $48 \mathrm{~h}($ ("P<0.05). (B) The proliferation of HT29 cells transfected with pcDNA3.1-miR-485-5p. Overexpression of miR-485-5p was significantly downregulated compared with the control group after $48 \mathrm{~h}(\mathrm{P}<0.05)$. (C) The proliferation of HCT8 cells transfected with pcDNA3.1-miR-485-5p. Overexpression of miR-485-5p was significantly downregulated compared with the control group after $72 \mathrm{~h}\left({ }^{*} \mathrm{P}<0.05\right)$. CRC, colorectal cancer.

A

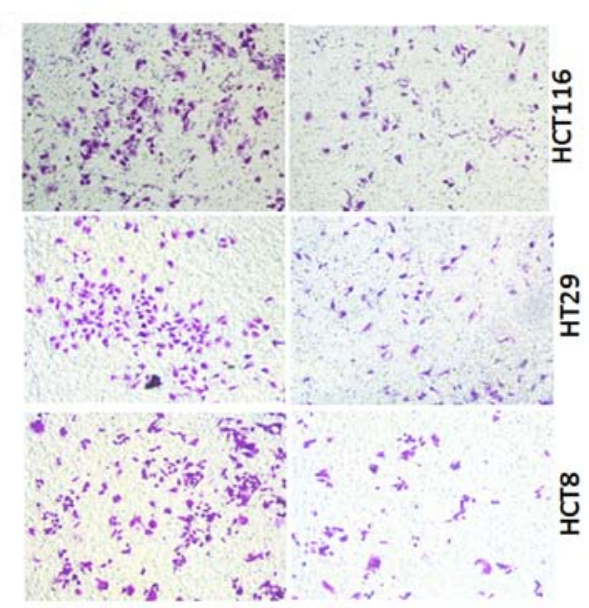

B

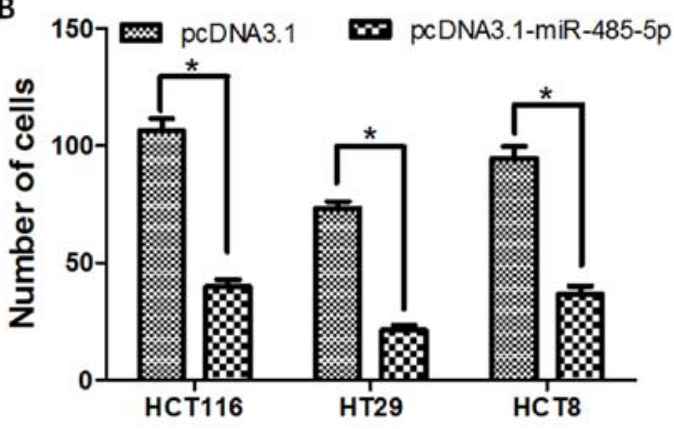

Figure 4. miR-485-5p suppresses cell invasion of CRC cells. (A) Representative images of the invasion of HCT116, HT29 and HCT8 cells transfected with pcDNA3.1 and pcDNA3.1-miR-485-5p are shown on the left panel (magnification, x200). (B) The number of cells that invaded through the chamber was evaluated in 3 fields for each experimental group and the average number of cells is presented on the right panel. "P<0.05. CRC, colorectal cancer.

A

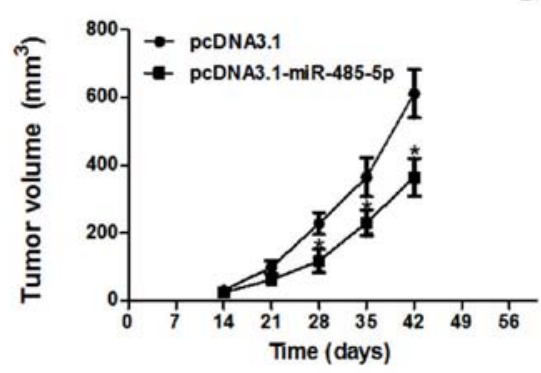

C

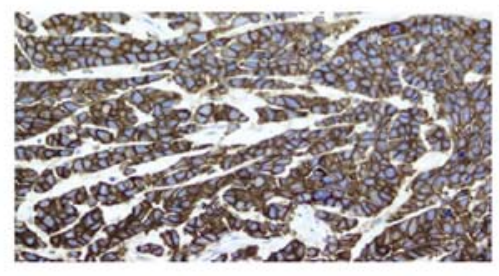

pcDNA3.1
B
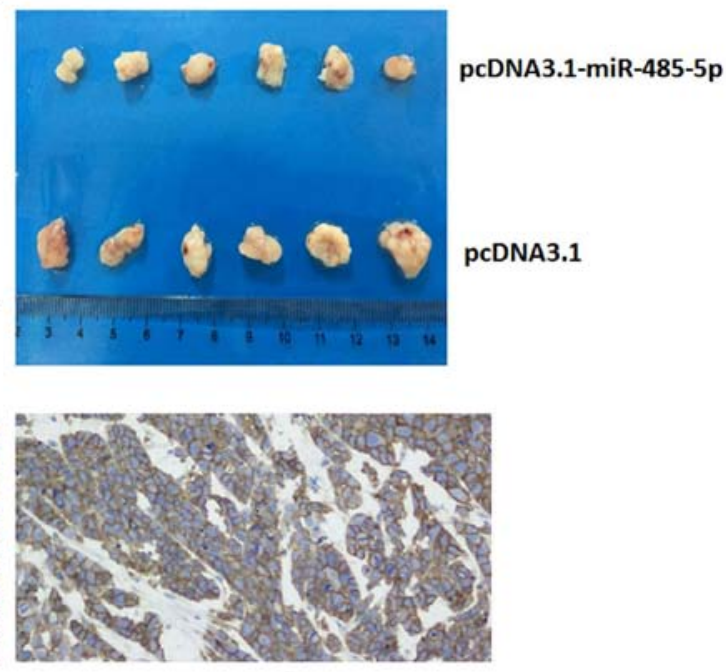

pcDNA3.1-miR-485-5p

Figure 5. Overexpression of miR-485-5p inhibits the tumor growth in HT29 xenograft models. (A) Tumor growth curves assessed after injection of HT29 cells stably transfected with pcDNA3.1-miR-485-5p or pcDNA3.1. The tumor volume was calculated once a week from week 2 to 6 . Values represent the means \pm SD for 6 mice/group. The tumor volume of the miR-485-5p overexpression groups was significantly decreased compared with the controls after 28 days. " $\mathrm{P}<0.05$. (B) Images of representative tumors in the control and miR-485-5p overexpression groups. (C) Immunohistochemical staining was performed in implanted tumors to detect the protein expression of CD147 (x400). 
A

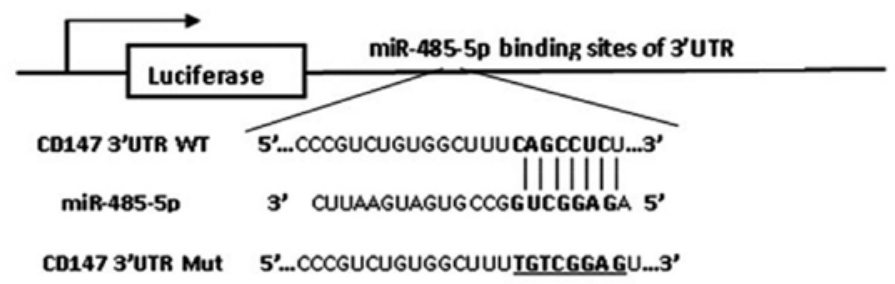

B

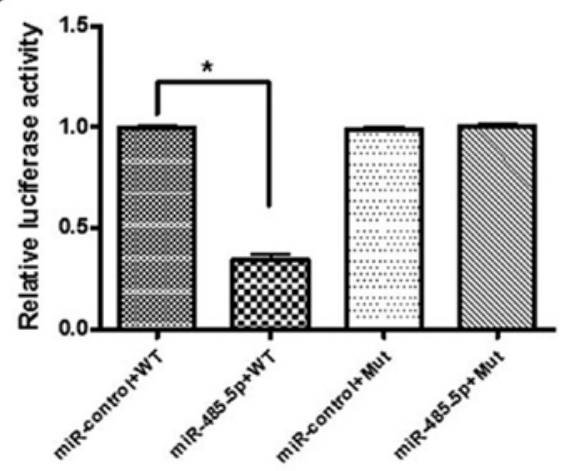

C

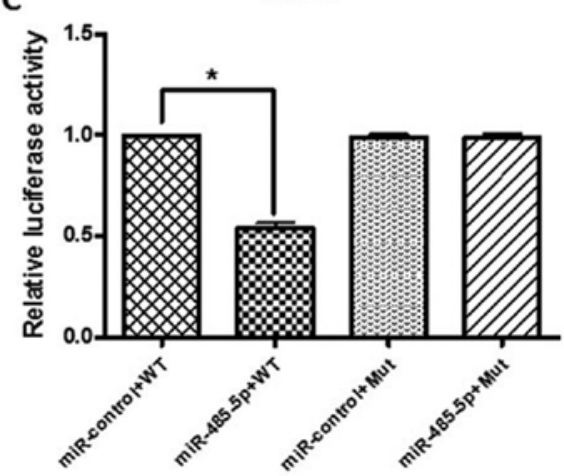

Figure 6. CD147 is a target gene of miR-485-5p. (A) The diagram of the luciferase reporter plasmids pcDNA3.1. The full length CD147 3'UTR was cloned into pmirGLO luciferase reporter, named CD147/3'UTR-WT. The mutant CD147 3'UTR of the miR-485-5p binding site was cloned into pmirGLO luciferase reporter, named CD147/3'UTR-Mut. (B) The CD147/3'UTR-WT and CD147/3'UTR-Mut were co-transfected into 293T cells with pcDNA3.1-miR-485-5p or pcDNA3.1-miR-control, and then the relative luciferase activity was determined. ${ }^{*} \mathrm{P}<0.05$. (C) The CD147/3'UTR-WT and CD147/3'UTR-Mut were co-transfected into HT29 cells with pcDNA3.1-miR-485-5p or pcDNA3.1-miR-control, and then the relative luciferase activity was determined. $\mathrm{P}<0.05$.

A

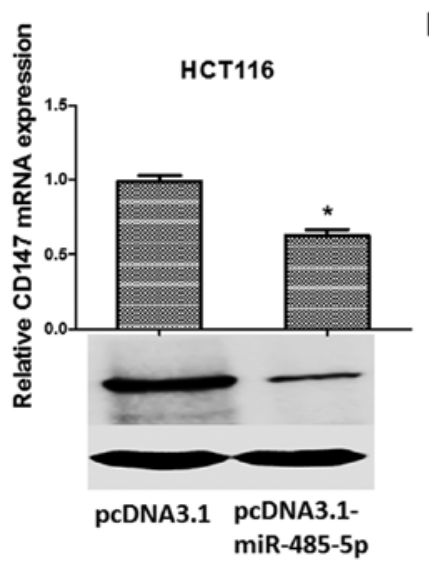

B

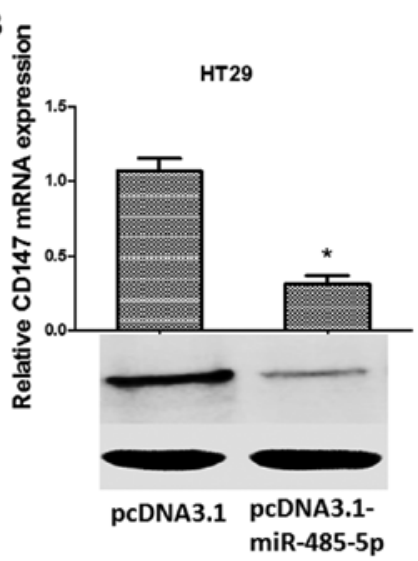

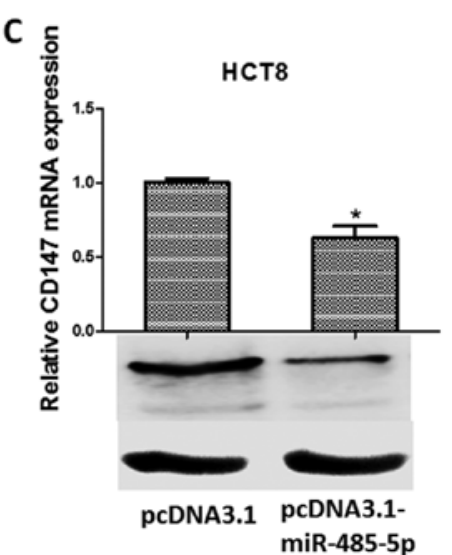

Figure 7. Experimental confirmation of the relationship between CD147 and miR-485-5p. (A) The expression of CD147 mRNA and protein in HCT116 cells transfected with pcDNA3.1-miR-485-5p was significantly downregulated compared with the pcDNA3.1 control groups, respectively $\left({ }^{*} \mathrm{P}<0.05\right)$. (B) The expression of CD147 mRNA and protein in HT29 cells transfected with pcDNA3.1-miR-485-5p was significantly downregulated compared with the pcDNA3.1 control groups, respectively ( $(\mathrm{P}<0.05)$. (C) The expression of CD147 mRNA and protein in HCT8 cells transfected with pcDNA3.1-miR-485-5p was significantly downregulated compared with the pcDNA3.1 control groups, respectively ( $\mathrm{P}<0.05)$.

CD147 is a target gene of $m i R-485-5 p$. We then investigated the mechanisms by which miR-485-5p inhibited CRC progression. Bioinformatics analysis revealed that the CD147 3'UTR was the target of miR-485-5p, so the CD147/3'UTR-WT and CD147/3'UTR-Mut with a substitution of nucleotides within the miR-485-5p binding site were constructed (Fig. 6A). The luciferase reporter assays were performed to ascertain whether miR-485-5p directly targeted CD147. Co-transfection of 293 T and HT29 cells with CD147/3'UTR-WT and pcDNA3.1-miR-485-5p caused a significant decrease in the luciferase activity compared with the control, and the luciferase activity of the CD147/3'UTR-Mut group was rescued
(Fig. 6B and $\mathrm{C}, \mathrm{P}<0.05$ ). The results confirmed that $\mathrm{CD} 147$ was the target gene of miR-485-5p in CRC cells.

miR-485-5p negatively regulates CD147 gene expression. In order to explore the relationship between CD147 and miR-485-5p, the expression of CD147 in transfected CRC cells was detected by RT-PCR and western blotting. The results revealed that overexpression of miR-485-5p in CRC cells significantly inhibited the expression of CD147 at the mRNA level (Fig. 7A-C, upper panels; $\mathrm{P}<0.05$ ) and protein level (Fig. 7A-C, lower panels). The results revealed that the upregulation of miR-485-5p could downregulate the expression of 


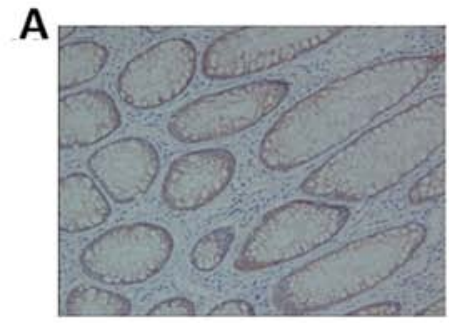

ANT

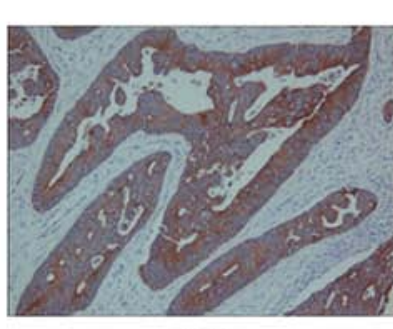

CRC

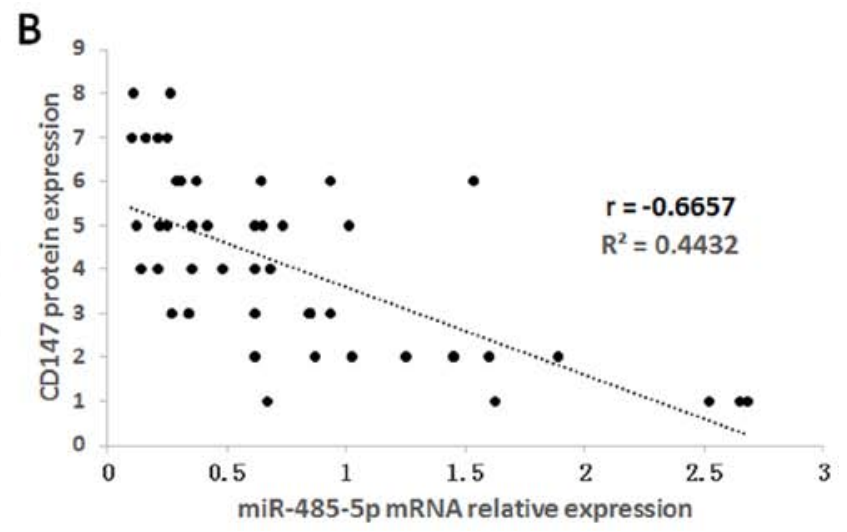

Figure 8. Correlation analyses between the CD147 protein and miR-485-5p expression. (A) Representative immunohistochemical staining for CD147 in CRC tissues and ANT tissues (x100). (B) The correlation analyses were performed between IHC scores of CD147 and the levels of miR-485-5p expression in CRC tissues. CRC, colorectal cancer; ANT, adjacent non-tumors; IHC, immunohistochemistry.

A

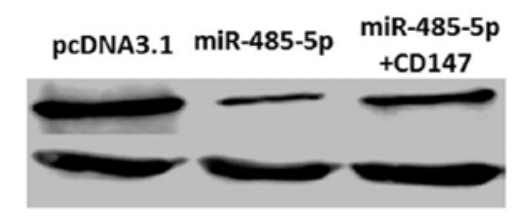

B

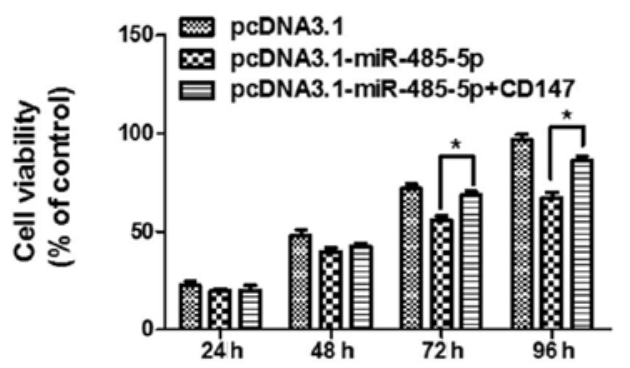

C

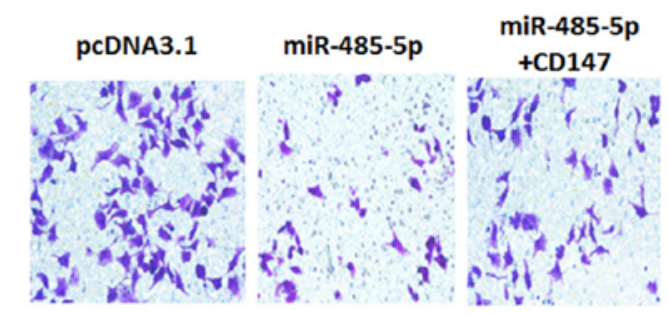

D

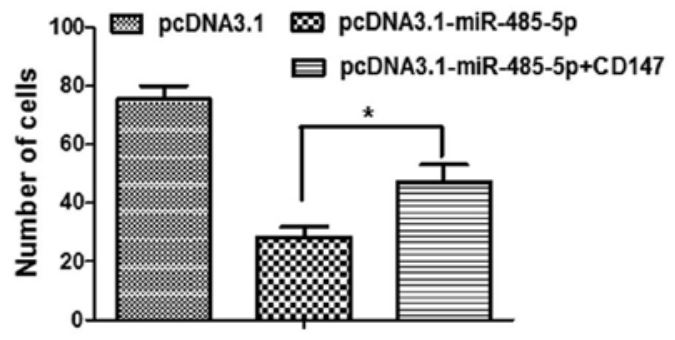

Figure 9. miR-485-5p regulates CRC cell proliferation and invasion by downregulating CD147. (A) Western blot analysis of CD147 expression in the miR-485-5p-overexpressing HT29 cell line after pcDNA3.1-CD147 transfection. (B) The proliferation of miR-485-5p overexpressing HT29 cells overexpressed with CD147 was significantly upregulated compared with the miR-485-5p overexpressing cells after $72 \mathrm{~h}\left({ }^{*} \mathrm{P}<0.05\right)$. (C) Representative images of the invasion of HT29 cells transfected with pcDNA3.1, pcDNA3.1-miR-485-5p and pcDNA3.1-miR-485-5p+pcDNA3.1-CD147 are displayed (magnification, $x 400$ ). (D) The number of cells that invaded through the chamber was evaluated in 3 fields for each experimental group and the average number of cells is presented. "P<0.05.

CD147. Moreover, the expression of the CD147 protein in forty-seven pairs of CRC and ANT tissues was detected by immunohistochemistry. The results revealed that the positive rate of CD147 was much higher in CRC (35/47) than that in ANT (15/47) (Fig. 8A). In addition, we detected the expression of miR-485-5p mRNA in CRC and ANT tissues by RT-PCR. The correlation analyses revealed that the expression of the CD147 protein was inversely correlated with the levels of miR-485-5p expression in CRC tissues (Fig. 8B), suggesting that miR-485-5p could negatively regulate CD147 expression in CRC tissues.

miR-485-5p regulates $C R C$ cell proliferation and invasion by downregulating CD147. In order to confirm that CD147 is the target gene of miR-485-5p, rescue experiments with CD147 in miR-485 overexpressed CRC cells were performed. We first constructed the CD147 expression vector pcDNA3.1-CD147, and co-transfected it with either the empty vector or pcDNA3.1-miR-485-5p in HT29 cells, and confirmed the efficiency of transfection by western blotting (Fig. 9A). Next, we performed a CCK-8 assay in HT29 cells, and the results indicated that $\mathrm{CD} 147$ overexpression partly reversed the inhibition of cell proliferation by miR- 485 overexpression (Fig. 9B). Furthermore, the invasion assay indicated that CD147 overexpression partly overcame the inhibition of cell invasion by miR-485-5p overexpression (Fig. 9C and D). Additionally, immunohistochemistry staining was performed in implanted tumors to detect the protein expression of CD147. As shown in Fig. 5C, the CD147 protein expression in the miR-485-5p overexpression group was significantly decreased compared with the control group. Collectively, the results revealed that CD147 downregulation by miR-485-5p contributed to the inhibition of CRC cell proliferation and invasion. 


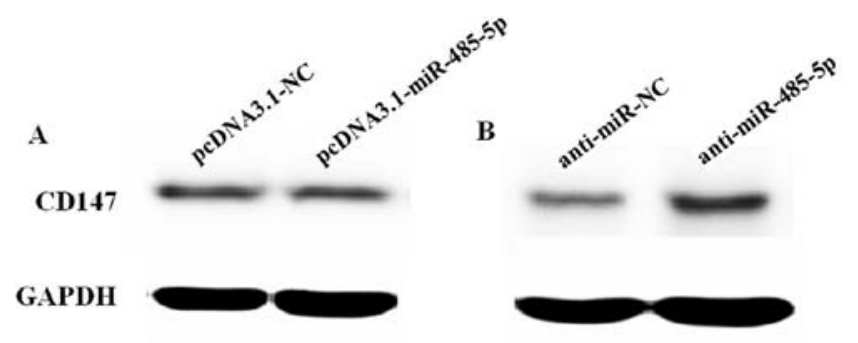

Figure 10. miR-485-5p regulates the expression of CD147 in FHC cells (A) Western blot analysis of CD147 expression in miR-485-5p overexpressing FHC cells after pcDNA3.1-CD147 transfection. (B) Western blot analysis of CD147 expression in miR-485-5p downregulated FHC cells after anti-miR-485-5p transfection.

Furthermore, we transfected pcDNA3.1-miR-485-5p into FHC cells (normal human intestinal epithelial cell line) to investigate the effects on CD147 expression. The results revealed that the expression of CD147 exhibited no significant alteration when the expression of miR-485-5p was increased (Fig. 10A). Then, we transfected anti-miR-485-5p into FHC cells. The results revealed that the expression of CD147 in cells transfected with anti-miR-485-5p was increased compared with the control (Fig. 10B).

\section{Discussion}

Colorectal cancer has become the third most common cancer in the US (1). In economically developing countries, the morbidity and mortality of CRC is rising, especially in China (21). There is great clinical value in identifying novel diagnostic or prognostic biomarkers that could improve the outcome of this disease.

Recently, research revealed that miRNAs may play important roles in the tumorigenesis and development of CRC. To date, the effect of miR-485-5p in human cancer has not been extensively reported. Recently, Chen et al revealed that miR-485-5p inhibited bladder cancer metastasis by targeting high mobility group AT-hook 2 (HMGA2) (11). Lou et al demonstrated that overexpression of miR-485-5p suppressed mitochondrial respiration and potential for cell migration and invasion in vitro, and also inhibited spontaneous metastasis of breast cancer cells in vivo. The suppression of mitochondrial respiration and cell invasion could be partially relieved by restoration of PGC-1 $\alpha$ expression, suggesting that miR-485-5p inhibited breast cancer cell migration and invasion by inhibiting PGC-1 $\alpha$ expression (22). Kang et al reported that upregulation of miR-485-5p could decrease the expression of Flot1 in gastric cancer cells, and ectopic expression of Flot1 partially reversed the inhibitory effect of enforced miR-485-5p expression on the malignant phenotypes of gastric cancer cells, suggesting that miR-485-5p could be a potential prognostic marker and function as a tumor suppressor in human gastric cancer by post-transcriptionally targeting Flot1 (10). Guo et al demonstrated that overexpression of miR-485-5p mimics could inhibit, while its antisense oligos promoted cell proliferation and invasion, and the dual-luciferase reporter gene assays and western blotting further revealed that stanniocalcin 2 was a direct target of miR-485-5p (23). In the present study, we demonstrated that the expression of miR-485-5p was lower in CRC tissues and cell lines than that in the normal controls. Overexpression of miR-485-5p in HCT116, HT29 and HCT8 cells significantly suppressed the proliferation and invasion capability of CRC cells in vitro. Moreover, overexpression of miR-485-5p could also inhibit the CRC tumor growth in vivo.

Previous studies found that the expression of CD147 was overexpressed in CRC and was associated with poor prognosis (17-19). In the present study, we used a dual luciferase system to ascertain that CD147 was the target gene of miR-485-5p. We found that CD147 mRNA and protein were suppressed when miR-485-5p was overexpressed in CRC cells. In addition, the expression of CD147 was negatively correlated to miR-485-5p in CRC tissues. Our results revealed that overexpression of miR-485-5p could inhibit the proliferation and invasion of CRC cells in vitro, and inhibit CRC tumor growth in vivo. In addition, CD147 contained the binding site for miR-485-5p, and overexpression of miR-485-5p could significantly suppress the expression of CD147.

The expression of CD147 in implanted tumors was detected by imm-unohistochemistry, and the results revealed that the CD147 protein expression in the miR-485-5p-overexpressed groups was significantly decreased compared with the control groups. The results revealed that overexpression of miR-485-5p may inhibit tumor growth by targeting CD147. In our previous study it was demonstrated that CD147 was highly expressed in CRC cells, and inhibition of CD147 expression reduced the ability of invasion in CRC cells (24). The possible mechanism involved was that CD147 silencing inhibited the secretion of MMPs. Moreover, CD147 silencing was able to increase the concentration of lactate thus leading to MCT1 protein reduction. In addition, the increase in lactate concentration may reduce cell growth or other tumor-associated biological activities.

In order to investigate the effects of anti-miR-485-5p on CRC cell proliferation and invasion, we transfected anti-miR-485-5p or anti-miR-NC into HT29 cells, respectively. However, the results revealed that the inhibition of miR-485-5p expression was not enough to affect the expression of CD147, and had no significant effect on cell proliferation and invasion (data not shown). Furthermore, we evaluated the effects of miR-485 overexpression and miR-485-5p inhibition on normal human intestinal epithelial cell line (FHC). The results revealed that simply increasing the expression of miR-485-5p did not significantly affect the expression of CD147. It is possible that the expression of CD147 was very low in normal cells. However, inhibition of miR-485-5p expression could increase the expression of CD147, suggesting that miR485-5p was an important regulator of CD147 expression. To the best of our knowledge, this is the first study to identify the function of miR-485-5p in CRC, and our results revealed that miR-485-5p may perform its effect partly through the inhibition of CD147.

In conclusion, miR-485-5p is downregulated in CRC, and miR-485-5p inhibits tumor development by targeting the CD147 gene in CRC. The miR-485-5p/CD147 link may provide novel diagnostic biomarkers and therapeutic strategies for CRC.

An in depth future study by the present authors will be performed continuing to investigate the functional mechanism 
of the miR-485-5p/CD147 link, and the diagnostic and prognostic values of circulating miR-485-5p in CRC patients.

\section{Acknowledgements}

Not applicable.

\section{Funding}

The present study was supported by a grant from the National Natural Science Foundation of China (no. 81501820) and the Jiangsu Youth Medical Talents Training Project to YP (QNRC2016074) and BH (QNRC2016066).

\section{Availability of data and materials}

The datasets used and/or analyzed during the current study are available from the corresponding author on reasonable request. All data generated or analyzed during this study are including in this published article.

\section{Authors' contributions}

SW and YP, study concept and design. HS, XH, BH, XL, TX, $\mathrm{XC}$ and $\mathrm{KZ}$, acquisition of data. YP, $\mathrm{HS}$ and $\mathrm{XH}$, analysis and interpretation of data. SW, YP and HS, drafting of the manuscript. All authors read and approved the final manuscript.

\section{Ethics approval and consent to participate}

The present study was approved by the Medical Ethics Committee of Nanjing First Hospital Affiliated to Nanjing Medical University, and all patients provided written informed consents.

\section{Consent for publication}

Not applicable.

\section{Competing interests}

The authors declare that they have no competing interests.

\section{Author's information}

SW acts as Technician/Professor/Vice President, Discipline leader and Director of Medical Laboratory Center in the Nanjing First Hospital, Nanjing, China.

\section{References}

1. Siegel RL, Miller KD and Jemal A: Cancer statistics, 2017. CA Cancer J Clin 67: 7-30, 2017.

2. Ciombor KK, Wu C and Goldberg RM: Recent therapeutic advances in the treatment of colorectal cancer. Annu Rev Med 66: 83-95, 2015.

3. Ren A, Dong Y, Tsoi $\mathrm{H}$ and Yu J: Detection of miRNA as non-invasive biomarkers of colorectal cancer. Int J Mol Sci 16 2810-2823, 2015.

4. Zeng CY, Zhan YS, Huang J and Chen YX: MicroRNA-7 suppresses human colon cancer invasion and proliferation by targeting the expression of focal adhesion kinase. Mol Med Rep 13: 1297-1303, 2016.
5. Slaby O: Non-coding RNAs as biomarkers for colorectal cancer screening and early detection. Adv Exp Med Biol 937: 153-170, 2016.

6. Jiang L, Cheng Q, Zhang BH and Zhang MZ: Circulating microRNAs as biomarkers in hepatocellular carcinoma screening: A validation set from China. Medicine (Baltimore) 94: e603, 2015.

7. Khoury S and Tran N: Circulating microRNAs: Potential biomarkers for common malignancies. Biomark Med 9: 131-151, 2015.

8. He N, Zheng H, Li P, Zhao Y, Zhang W, Song F and Chen K: miR-485-5p binding site SNP rs8752 in HPGD gene is associated with breast cancer risk. PLoS One 9: e102093, 2014.

9. Kim TH, Kim YK, Kwon Y, Heo JH, Kang H, Kim G and An HJ: Deregulation of miR-519a, 153, and 485-5p and its clinicopathological relevance in ovarian epithelial tumours. Histopathology 57: 734-743, 2010.

10. Kang M, Ren MP, Zhao L, Li CP and Deng MM: miR-485-5p acts as a negative regulator in gastric cancer progression by targeting flotillin-1. Am J Transl Res 7: 2212-2222, 2015.

11. Chen Z, Li Q, Wang S and Zhang J: miR-485-5p inhibits bladder cancer metastasis by targeting HMGA2. Int J Mol Med 36: 1136-1142, 2015.

12. Rosenthal EL, Shreenivas S, Peters GE, Grizzle WE, Desmond R and Gladson CL: Expression of extracellular matrix metalloprotease inducer in laryngeal squamous cell carcinoma. Laryngoscope 113: 1406-1410, 2003.

13. Gallagher SM, Castorino JJ, Wang D and Philp NJ: Monocarboxylate transporter 4 regulates maturation and trafficking of CD147 to the plasma membrane in the metastatic breast cancer cell line MDA-MB-231. Cancer Res 67: 4182-4189, 2007.

14. Sato M, Nakai Y, Nakata W, Yoshida T, Hatano K, Kawashima A, Fujita K, Uemura M, Takayama H and Nonomura N: EMMPRIN promotes angiogenesis, proliferation, invasion and resistance to sunitinib in renal cell carcinoma, and its level predicts patient outcome. PLoS One 8: e74313, 2013.

15. Omi Y, Shibata N, Okamoto T, Obara T and Kobayashi M: The role of CD147 in the invasiveness of follicular thyroid carcinoma cells. Thyroid 22: 383-394, 2012.

16. Stenzinger A, Wittschieber D, von Winterfeld M, Goeppert B, Kamphues C, Weichert W, Dietel M, Rabien A and Klauschen F: High extracellular matrix metalloproteinase inducer/CD147 expression is strongly and independently associated with poor prognosis in colorectal cancer. Hum Pathol 43: 1471-1481, 2012.

17. Zhu S, Chu D, Zhang Y, Wang X, Gong L, Han X, Yao L, Lan M, Li Y and Zhang W: EMMPRIN/CD147 expression is associated with disease-free survival of patients with colorectal cancer. Med Oncol 30: 369, 2013.

18. Zheng HC, Wang W, Xu XY, Xia P, Yu M, Sugiyama T and Takano Y: Up-regulated EMMPRIN/CD147 protein expression might play a role in colorectal carcinogenesis and its subsequent progression without an alteration of its glycosylation and mRNA level. J Cancer Res Clin Oncol 137: 585-596, 2011.

19. Pan Y, He B, Song G, Bao Q, Tang Z, Tian F and Wang S: CD147 silencing via RNA interference reduces tumor cell invasion, metastasis and increases chemosensitivity in pancreatic cancer cells. Oncol Rep 27: 2003-2009, 2012.

20. Pan Y, He B, Chen J, Sun H, Deng Q, Wang F, Ying H, Liu X, Lin K, Peng H, et al: Gene therapy for colorectal cancer by adenovirus-mediated siRNA targeting CD147 based on loss of the IGF2 imprinting system. Int J Oncol 47: 1881-1889, 2015.

21. Gray RT, Coleman HG, Hughes C, Murray LJ and Cardwell CR: Statin use and survival in colorectal cancer: Results from a population-based cohort study and an updated systematic review and meta-analysis. Cancer Epidemiol 45: 71-81, 2016.

22. Lou C, Xiao M, Cheng S, Lu X, Jia S, Ren Y and Li Z: miR-485-3p and miR-485-5p suppress breast cancer cell metastasis by inhibiting PGC-1 $\alpha$ expression. Cell Death Dis 7: e2159, 2016.

23. Guo GX, Li QY, Ma WL, Shi ZH and Ren XQ: MicroRNA-485-5p suppresses cell proliferation and invasion in hepatocellular carcinoma by targeting stanniocalcin 2. Int J Clin Exp Pathol 8: 12292-12299, 2015.

24. Li R, Pan Y, He B, Xu Y, Gao T, Song G, Sun H, Deng Q and Wang S: Downregulation of CD147 expression by RNA interference inhibits HT2 9 cell proliferation, invasion and tumorigenicity in vitro and in vivo. Int J Oncol 43: 1885-1894, 2013. 